\title{
Considered view of Neptune
}

\section{Washington}

THE spectacular shot, shown below, of Neptune's ring system was taken by Voyager 2 as it looked back towards the planet. In sunlight reflected at a glancing angle, the fainter rings and dust bands are clearly visible, but a comparison with pictures of the rings taken as Voyager approached Neptune (see Nature of 31 August) shows an intriguing difference: the outermost ring, significantly brighter on the approach shots, shows up here a little fainter than the prominent inner ring.

The difference is a clue to the composition of the rings. Particles bigger than the wavelength of light, about half a micrometre, scatter most of the radiation incident upon them directly backwards, towards the light source; smaller particles scatter more light forwards than backwards. Thus most of the ring and disk material around Neptune, except for the outer ring, is 'dust' - although a more apt comparison for the size of the particles would be 'smoke'.

An extended disk of fine material was presaged by another Voyager experiment. The plasma-wave detector, two long antennas arranged in a V, also acts as a dust detector. As Voyager 2 speeds on at several miles a second, any dust particles it encounters vaporize on impact: the ionized shock front from this tiny explosion carries a characteristic burst of radio noise, which the plasma antenna picks up.

Because the frequency of the radio noise from the dust impacts is in the kilohertz range, it can be replayed directly as an audio signal: this led to a moment of

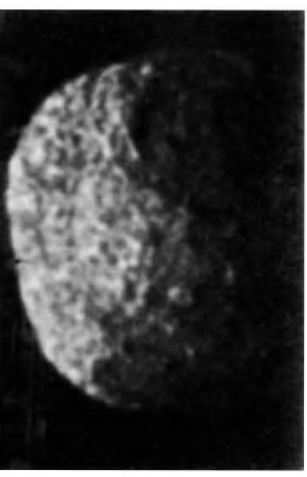

The largest of Neptune's 'new' moons, 1989N1, turned out to be oddly shaped. At about $250 \mathrm{~km}$ in radius, and thought to be made of deep-frozen water ice, it could not be any bigger without its own gravity forcing it into a spherical shape. Below, the rings in their full splendour, seen as Voyager looked backwards after passing by Neptune.

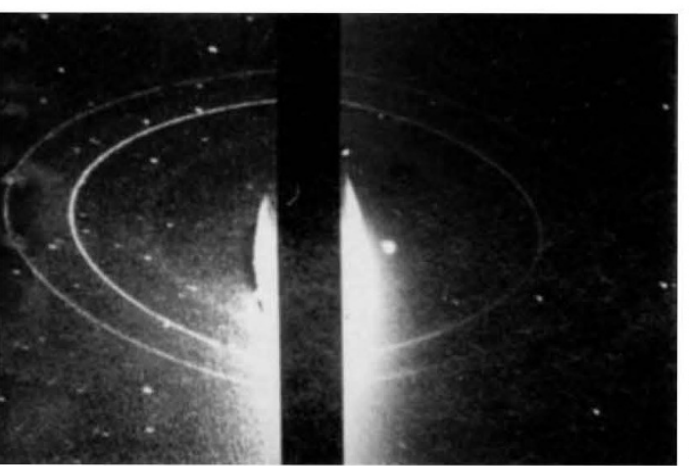

NATURE · VOL $341 \cdot 14$ SEPTEMBER 1989 drama in the Neptune encounter. As Voyager 2 passed through the equatorial plane on its way to closest approach, at about twice the radial distance of the outer ring, the hissing and crashing of the dust was played live through loudspeakers at the Jet Propulsion Laboratory. As the noise reached a maximum, it abruptly ceased; Voyager's radio signal was lost behind Neptune, to be recovered 45 minutes later.

Calculations of the encounter rate with dust around Neptune's equator suggested a maximum density of 0.003 particles per cubic metre. This is more dust than found around Uranus, and its presence, along with the 'clumpy' outer ring, reinforces the idea that Neptune has had a more interesting recent history than its sister planet. Explaining the denser segments in the outer ring will be the hardest task: two theories already on the books both seem to be misses. In the first, two guiding satellites, one at the same orbital radius as the ring and one just inside, create perturbations to the gravitational field that allow material to reside stably in restricted arcs; at Neptune, none of the four new satellites near the ring is in the right place. In the second theory, a satellite with an orbit inclined to the ring plane creates similar perturbations, and 1989N6 is indeed in a circular orbit tilted at 5 degrees. But it seems too small for its gravitational influence to be able to stabilize the dense ring segments.

In another respect, Neptune and Uranus are twins: both have magnetic fields whose axis is tilted, at about 50 and 60 degrees respectively, markedly from the rotation axis. This presumably has something to do with the internal structure of the planet. Unlike Earth, which has a hot, semiliquid core whose internal motions generate the terrestrial magnetic field, the rocky cores of the giant planets are small, cold and inert. Outside this core there is probably a thick layer of liquid water existing, because of the huge pressure, in an ionized phase. The dynamo for these planets may reside in this turgid but mobile region.

The tilted magnetic field complicates Neptune's magnetosphere, and its interaction with the solar wind. Detectors on Voyager found evidence for charged particles trapped by the magnetic field, but the density was unexpectedly low. The magnetosphere, whose geometry is con-
A false-colour composite of UV, violet and green reveals the altitude of Neptune's clouds. The bright pink cirrus clouds are above most of the atmosphere, which absorbs UV radiation and darkens the deeper clouds. The Great Dark Spot appears blue; it lies at an intermediate height from which blue light, but not UV, can escape.
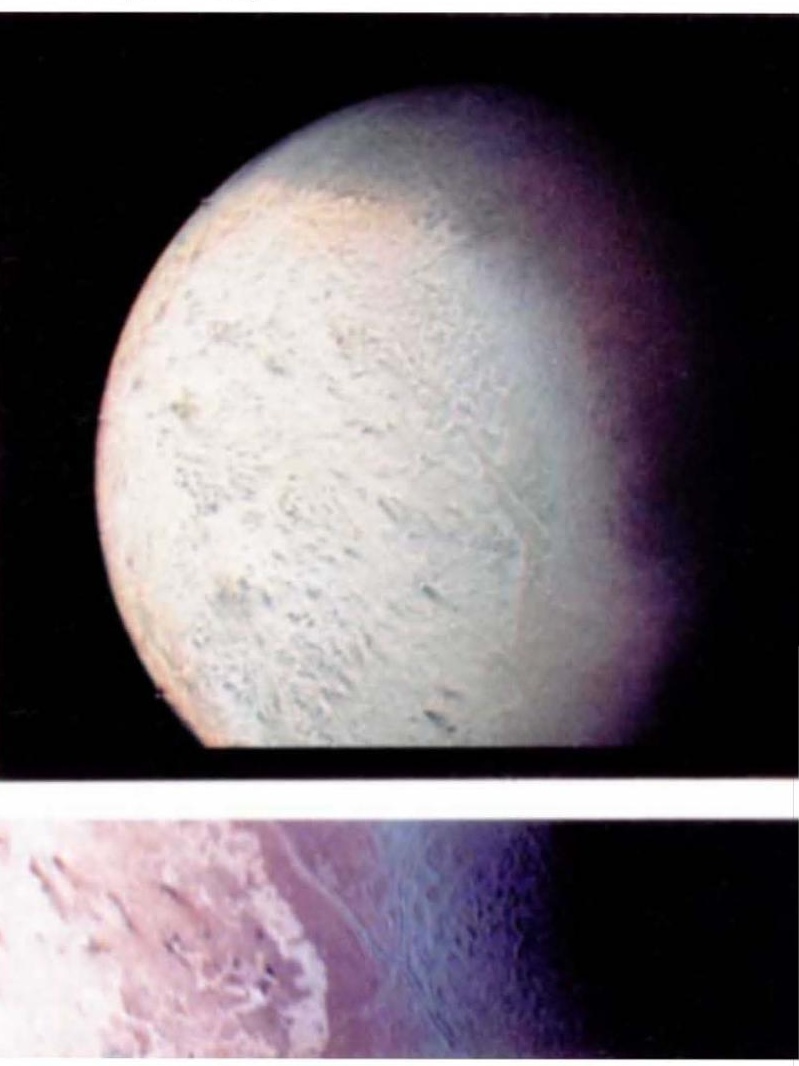

A false-colour view of Triton, taken on Voyager's approach. The pink hue approximates to the real colour of methane ice, but the blue represents UV light: sadly, Triton is not a blue moon. The strip below, a composite of several shots, shows the variation in terrain. The feeble heat of Triton's summer is thought to sublimate surface ices, breaking up the smooth surface into the blotchy covering at left.

trolled by a tilted magnetic pole rotating about a planetary axis itself tilted with respect to the plane of the Solar System, may not be permanently isolated from the solar wind: from time to time, particles trapped within it can be swept out into interplanetary space.

David Lindley 